\title{
Disseminated Histoplasmosis in an Immunocompetent Patient After COVID-19 Pneumonia
}

\author{
Matthew Taylor ${ }^{1}$, Arjun Ghodasara ${ }^{1}$, Ali Ismail ${ }^{2}$, Umair Gauhar ${ }^{3}$, Karim El-Kersh ${ }^{4}$ \\ 1. Pulmonary and Critical Care, University of Louisville, Louisville, USA 2. Pathology and Laboratory Medicine, \\ University of Louisville, Louisville, USA 3. Interventional Pulmonary, University of Louisville, Louisville, USA 4. \\ Pulmonary and Critical Care, University of Nebraska Medical Center, Omaha, USA
}

Corresponding author: Matthew Taylor, matt.j.taylor14@gmail.com

\begin{abstract}
Disseminated histoplasmosis can occur in immunocompromised patients such as in HIV disease and patients with medication-induced immunosuppression. Most of these patients present with fever, weight loss, hepatosplenomegaly, lymphadenopathy, and pancytopenia. There are increasing reports of coronavirus disease 2019 (COVID-19) pneumonia associated with fungal infections including aspergillus and mucormycosis. It is not typical for immunocompetent patients to present with disseminated fungal disease. We herein report a case of a 50-year-old immunocompetent male with a recent recovery from COVID-19 pneumonia who presented with fever and pancytopenia. Chest computed tomography (CT) demonstrated new-onset right upper lobe lung mass, subcarinal lymphadenopathy, and splenomegaly. Mediastinal lymph nodes and bone marrow biopsies were performed, and the patient was diagnosed with disseminated histoplasmosis. The association between COVID-19 pneumonia and fungal infections is increasingly reported. Diagnosis requires a high index of suspicion, especially in immunocompetent patients.
\end{abstract}

Categories: Pathology, Infectious Disease, Pulmonology

Keywords: covid-19, disseminated histoplasmosis, fungal, immunocompetent, dexamethasone

\section{Introduction}

Coronavirus disease 2019 (COVID-19) pneumonia-associated fungal infections are well documented with risk factors including ICU prolonged length of stay, neutropenia, hematological malignancies treated with chemotherapy, transplantation, and other immunocompromised states like HIV [1,2]. Disseminated histoplasmosis is often seen in immunosuppressed patients, and can present with multisystem involvement including the lungs, skin, gastrointestinal tract, and bone marrow [3-5]. Disseminated histoplasmosis in patients with post-COVID-19 pneumonia is rare and is difficult to diagnose [6]. A disseminated disease requires treatment and with amphotericin B for one to two weeks followed by azole antifungal therapy [7].

Review began 07/05/2021 Review ended 08/13/2021 Published 08/18/2021

\section{(c) Copyright 2021}

Taylor et al. This is an open access article distributed under the terms of the Creative Commons Attribution License CC-BY 4.0., which permits unrestricted use, distribution, and reproduction in any medium, provided the original author and source are credited.

\section{Case Presentation}

A 50-year-old male that lived in the Ohio River Valley with a history of mild intermittent asthma well controlled on albuterol and fluticasone-salmeterol and no recent requirement of oral corticosteroids was admitted for daily cyclical fevers after COVID-19 pneumonia. One month before admission, the patient was treated on the medical-surgical floor for COVID-19 pneumonia with a five-day course of remdesivir and a 10-day course of dexamethasone. The patient recovered and he was discharged home without oxygen. Two weeks after discharge, he developed fever and myalgias. The patient had two polymerase chain reaction (PCR) COVID-19 testing that both were negative. On hospital admission, COVID-19 PCR testing was also negative. His blood work on admission revealed WBC 7.9 x $10^{3} \mathrm{uL}$, hemoglobin $11.8 \mathrm{~g} / \mathrm{dL}$, and platelets $146 \mathrm{x}$ $10^{3} \mathrm{uL}$. A non-contrast chest computed tomography (CT) revealed interval improvement of ground-glass opacities, a new $3.9 \mathrm{~cm}$ mass-like density in the right upper lobe (RUL), mildly enlarged mediastinal lymphadenopathy, and moderate-severe hepatosplenomegaly (Figures 1,2). The patient was started on vancomycin and cefepime and transitioned to linezolid, levofloxacin, voriconazole, and piperacillintazobactam after no improvement in his fevers as high as $39.9^{\circ} \mathrm{C}$. The patient continued to be febrile daily until hospital day 14 despite antipyretics. He was first noted to have pancytopenia on hospital day 7 with a possible cause due to Linezolid which was stopped on hospital day six. His repeat blood work revealed WBC $2.8 \times 10^{3} \mathrm{uL}$, hemoglobin $8.8 \mathrm{~g} / \mathrm{dL}$, platelets $50 \times 10^{3} \mathrm{uL}$, and ferritin 2,934 ng/mL. The patient underwent bronchoscopy with endobronchial ultrasound and transbronchial needle aspiration of subcarinal lymph node and biopsy of the RUL mass. Fine needle aspiration of the subcarinal lymph node showed budding yeast suggestive of histoplasmosis on the Grocott methenamine silver (GMS) stain (Figure 3). The Histoplasma galactomannan urine antigen was positive at $1.6 \mathrm{ng} / \mathrm{mL}$ (reference range $<0.2 \mathrm{ng} / \mathrm{mL}$ ). The patient's serum cryptococcal antigen and antibody and blastomyces antibody were negative. The bronchial alveolar lavage and tissue biopsy of the right upper lobe mass isolated Histoplasma capsulatum. Bone marrow biopsy revealed extensive granulomatous inflammation consistent with disseminated 


\section{Cureus}

histoplasmosis. His cyclic fevers ceased, and pancytopenia improved with amphotericin-B. He was discharged from the hospital one week after starting treatment with a plan of two weeks of therapy and transition to oral fluconazole.

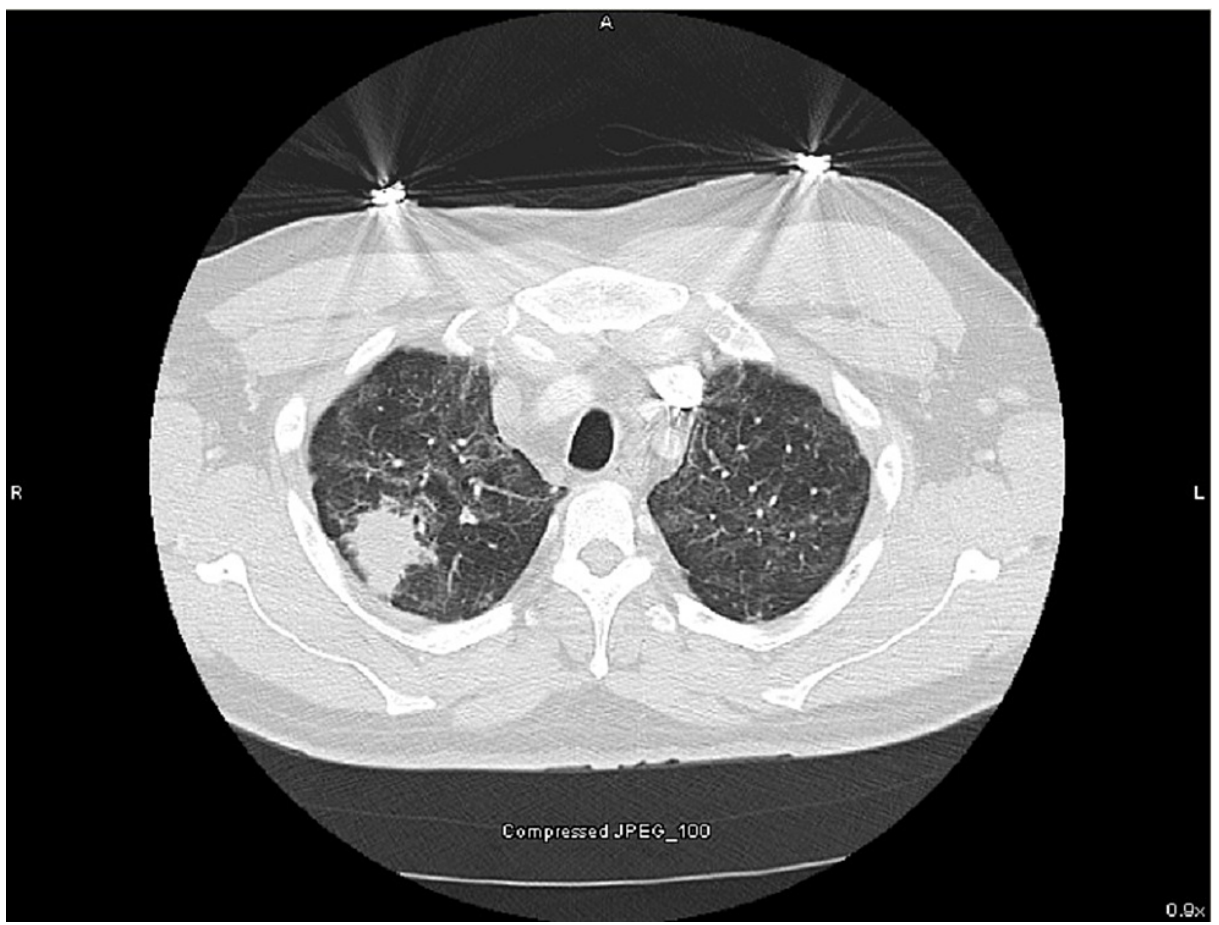

FIGURE 1: CT of the chest with right upper lobe (RUL) mass.

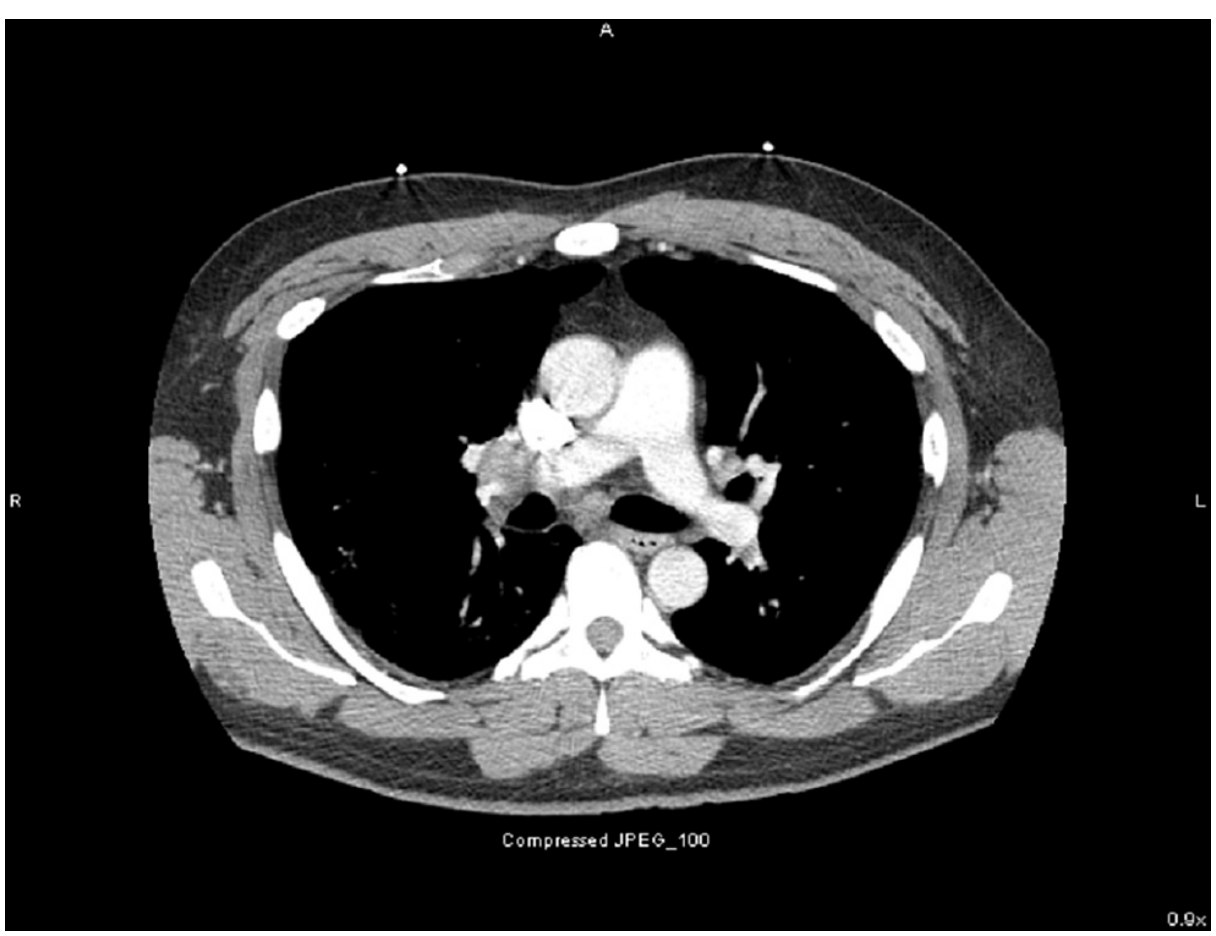

FIGURE 2: CT of the chest with subcarinal lymph node enlargement. 


\section{Cureus}

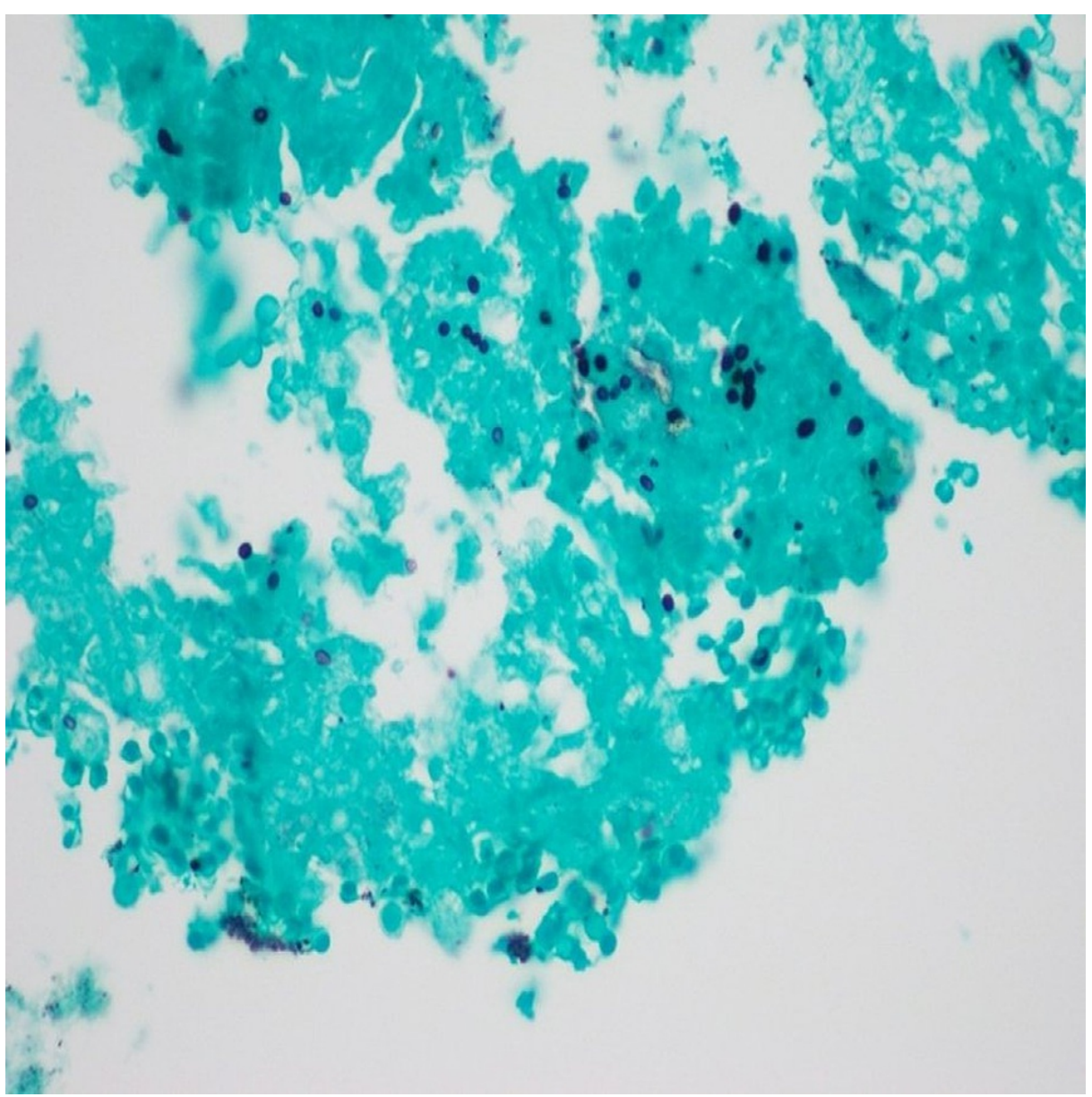

FIGURE 3: Grocott methenamine silver (GMS) stain with budding yeast.

\section{Discussion}

Risk factors for most of the reported cases of disseminated histoplasmosis in immunocompetent patients include living in a rural setting due to the likely exposure to histoplasma in the soil [8]. Most of these patients experienced fever, weight loss, hepatosplenomegaly, lymphadenopathy, pancytopenia, elevated ESR, elevated hepatic transaminases, and had bone marrow involvement [8,9]. An immunocompromised state, including HIV/AIDS and chemotherapy-induced immunosuppression, is a known risk factor for disseminated histoplasmosis. There is a reported case of disseminated histoplasmosis in a patient with HIV/AIDS after COVID-19. This patient presented with an initial CD4 count of 113 cells $/ \mathrm{mm}^{3}$ and a viral load of 38,503 RNA copies/ml. One week after the initial presentation, the patient was diagnosed with COVID-19 and with suspected disseminated histoplasmosis based on a positive histoplasmosis antigen and CT scan with lymphadenopathy and hepatosplenomegaly [6]. Another patient with underlying asthma and diabetes mellitus presented with COVID-19 pneumonia complicated by pulmonary embolism and prolonged hospitalization was diagnosed with pulmonary histoplasmosis based on positive Histoplasma capsulatum complement fixation titers [10].

Co-infecting pathogens with COVID-19 are well documented, and patients are more likely to die than those without a co-infection [2]. Initial reports included both viral and bacterial causes [2,11]. Fungal co-infections are increasingly reported after COVID-19 and are also known to increase mortality as well [12]. Most reported cases include aspergillus with a prevalence as high as $8.5 \%$ in retrospective studies and $9 \%$ in a meta-analysis [2]. Risk factors not mentioned previously for aspergillosis include antibiotics, particularly azithromycin (cumulative dose $>/=1500 \mathrm{mg}$ ) [13]. Many of these patients may be treated with Voriconazole to avoid the potentially nephrotoxic liposomal amphotericin B [12]. Candida, which is often isolated in the respiratory tract, is usually not thought to be pathogenic [1,2] and the reported cases of candida bacteremia were thought to be related to central line infections [14]. Other reported non-disseminated fungal coinfections include coccidioidomycosis, mucormycosis, and cryptococcosis $[1,11]$.

Acute pulmonary histoplasmosis presents with either asymptomatic disease, flu-like symptoms, caseating or noncaseating granulomatous disease, or mediastinal adenopathy. Chronic pulmonary histoplasmosis presents with an either cavitary or noncavitary disease with associated nodules, infiltrates, and mediastinal lymphadenopathy. Disseminated disease is often seen in immunosuppressed patients. Manifestations can 
include skin involvement with polymorphic plaques, nodules, and erosions, gastrointestinal involvement with hepatosplenomegaly and colonic ulcerations, and bone marrow suppression $[3,4]$.

Diagnosis of disseminated disease is complex as many tests take weeks. Cultures often take two to four but up to eight weeks with the greatest yield occurring in those with disseminated disease. Complement fixing antibodies may appear two to six weeks following acute infection and may persist for years following infection. Immunodiffusion antibody assay tests for the presence of $\mathrm{M}$ and $\mathrm{H}$ precipitin bands. $\mathrm{M}$ bands develop with acute infections and persist for months to years. $\mathrm{H}$ bands appear after an $\mathrm{M}$ band and may disappear earlier than $\mathrm{M}$ bands. These may indicate active histoplasmosis [3]. Antigen testing may be more effective in acute disease and immunocompromised patients who cannot amount an antibody response with improved sensitivity utilizing both serum and urine testing. A polymerase chain reaction is available but still needs further standardization $[3,15]$.

Treatment for the mild asymptomatic acute pulmonary disease is often unnecessary. Moderate to severe acute pulmonary disease requires treatment with amphotericin B followed by azole antifungal therapy for weeks. Chronic pulmonary disease is treated with azole antifungal therapy for 12-24 months. The disseminated disease requires treatment with amphotericin B for one to two weeks followed by azole antifungal therapy for at least 12 months [7].

\section{Conclusions}

We presented a case of disseminated histoplasmosis after COVID-19 pneumonia effectively treated with amphotericin-B and fluconazole. The patient was eventually discharged home with resolution of his cyclic fevers and pancytopenia. Fungal infections are increasingly reported in association with COVID-19 pneumonia. Although not able to prove, prolonged courses of dexamethasone may be a risk factor for disseminated fungal infections. Clinicians need to be aware of the possibility of disseminated fungal diseases even in immunocompetent patients.

\section{Additional Information \\ Disclosures}

Human subjects: Consent was obtained or waived by all participants in this study. The University of Louisville Institutional Review Board issued approval 727224. The IRB Chair/Vice-Chair (or An IRB member) has reviewed your submission. The project described does not meet the "Common Rule" definition of human subjects' research. The IRB has classified this project as Non-Human Subjects Research (NHSR). The project can proceed. Conflicts of interest: In compliance with the ICMJE uniform disclosure form, all authors declare the following: Payment/services info: All authors have declared that no financial support was received from any organization for the submitted work. Financial relationships: Karim El-Kersh declare(s) personal fees from United Therapeutics, Actelion, Acceleron Pharma. Speaker, Consultant (advisory board) for United Therapeutics. Consultant (advisory board) for Actelion. Consultant for Acceleron Pharma. . Other relationships: All authors have declared that there are no other relationships or activities that could appear to have influenced the submitted work.

\section{Acknowledgements}

Figure 2 provided by Dr. Ali Ismail MBBS, Department of Pathology and Laboratory Medicine, University of Louisville School of Medicine 2021.

\section{References}

1. Song G, Liang G, Liu W: Fungal co-infections associated with global COVID-19 pandemic: a clinical and diagnostic perspective from China. Mycopathologia. 2020, 185:599-606. 10.1007/s11046-020-00462-9

2. Lansbury L, Lim B, Baskaran V, Lim WS: Co-infections in people with COVID-19: a systematic review and meta-analysis. J Infect. 2020, 81:266-75. 10.1016/j.jinf.2020.05.046

3. Araúz AB, Papineni P: Histoplasmosis. Infect Dis Clin North Am. 2021, 35:471-91. 10.1016/j.idc.2021.03.011

4. Mussá NY, Ismail S, Carvalho D: Disseminated histoplasmosis diagnosed in a bone marrow sample [Epub ahead of print]. Hematol Transfus Cell Ther. 2020, 10.1016/j.htct.2020.10.960

5. Sumiyoshi S, Tanaka S, Kato H, et al.: Diagnosis by molecular pathology of an early and atypical histoplasmosis lesion in the duodenum of an immunocompromised patient: a case report. Biomed Rep. 2021, 14:6. 10.3892/br.2020.1382

6. Basso RP, Poester VR, Benelli JL, et al.: COVID-19-associated histoplasmosis in an AIDS patient . Mycopathologia. 2021, 186:109-12. 10.1007/s11046-020-00505-1

7. Wheat LJ, Freifeld AG, Kleiman MB, Baddley JW, McKinsey DS, Loyd JE, Kauffman CA: Clinical practice guidelines for the management of patients with histoplasmosis: 2007 update by the Infectious Diseases Society of America. Clin Infect Dis. 2007, 45:807-25. 10.1086/521259

8. De D, Nath UK: Disseminated Histoplasmosis in Immunocompetent Individuals- not a so Rare Entity, in India. Mediterr J Hematol Infect Dis. 2015, 7 :e2015028. 10.4084/MJHID.2015.028

9. Dang Y, Jiang L, Zhang J, et al.: Disseminated histoplasmosis in an immunocompetent individual diagnosed with gastrointestinal endoscopy: a case report. BMC Infect Dis. 2019, 19:992. 10.1186/s12879-019-4542-X

10. Cafardi J, Haas D, Lamarre T, Feinberg J: Opportunistic fungal infection associated with COVID-19. Open 


\section{Cureus}

Forum Infect Dis. 2021, ofab016. 10.1093/ofid/ofab016

11. Shah AS, Heidari A, Civelli VF, et al.: The coincidence of 2 epidemics, coccidioidomycosis and SARS-CoV-2: a case report. J Investig Med High Impact Case Rep. 2020, 8: 10.1177/2324709620930540

12. Hoenigl M: Invasive fungal disease complicating COVID-19: when it rains it pours [Epub ahead of print] . Clin Infect Dis. 2020, 10.1093/cid/ciaa1342

13. Dellière S, Dudoignon E, Fodil S, et al.: Risk factors associated with COVID-19-associated pulmonary aspergillosis in ICU patients: a French multicentric retrospective cohort. Clin Microbiol Infect. 2020, 10.1016/j.cmi.2020.12.005

14. Hughes S, Troise O, Donaldson H, Mughal N, Moore LS: Bacterial and fungal coinfection among hospitalized patients with COVID-19: a retrospective cohort study in a UK secondary-care setting. Clin Microbiol Infect. 2020, 26:1395-9. 10.1016/j.cmi.2020.06.025

15. Vidal JE, Werlang PC, Muniz BM, et al.: Combining urine antigen and blood polymerase chain reaction for the diagnosis of disseminated histoplasmosis in hospitalized patients with advanced HIV disease [Epub ahead of print]. Med Mycol. 2021, 10.1093/mmy/myab022 